\title{
Neuropatia auditiva: alerta aos pediatras
}

\author{
Auditory neuropathy: alert to pediatricians
}

Flávia Varela Capone ${ }^{1}$, Danielle de Araújo Torres ${ }^{1}$, Marco Antonio de M. T. de Lima ${ }^{2}$

\section{RESUMO}

Objetivo: Alertar os pediatras sobre a neuropatia auditiva, doença descrita recentemente e ainda desconhecida por muitos médicos. Descrever seus fatores de risco, características clínicas e diagnósticas, com a finalidade de possibilitar uma intervenção terapêutica precoce e eficaz.

Fontes de dados: Realizada pesquisa nas bases de dados PubMed, Lilacs e SciELO utilizando os descritores "neuropatia auditiva" e "auditory neuropatby", entre os anos de 1996 e 2010 .

Síntese dos dados: A neuropatia auditiva, também conhecida como dessincronia auditiva, descrita em 1996, caracteriza-se clinicamente pela dificuldade na compreensão das palavras, mesmo em casos de perdas auditivas leves ou moderadas. Foi relacionada a diversas neuropatias generalizadas e fatores de risco neonatais, como internação em terapia intensiva, hiperbilirrubinemia, sepse e hipóxia. Após suspeita clínica, o diagnóstico é confirmado pela presença das emissões otoacústicas associada a um potencial evocado auditivo de tronco encefálico ausente ou alterado. Sua terapêutica permanece controversa, tendo como opções a protetização auditiva, o acompanhamento fonoterápico para habilitação ou reabilitação da linguagem e, em casos de insucesso, há relatos de resultados satisfatórios com o implante coclear.

Conclusões: Enfatiza-se a importância do reconhecimento pelo pediatra da neuropatia auditiva, entidade ainda pouco citada na literatura latino-americana da especialidade.

Palavras-chave: perda auditiva neurossensorial; testes auditivos; triagem neonatal.

\section{ABSTRACT}

Objective: To alert pediatricians about the auditory neuropathy, a disease that has been only recently described and is still unknown by many physicians, and to describe its risk factors, clinical and diagnostic features in order to enable an early and effective therapeutic intervention.

Data source: A literature search using terms such as "auditory neuropathy" and "neuropatia auditiva" has been conducted in the PubMed, Lilacs and SciELO databases between 1996 and 2010.

Data synthesis: Described in 1996, the auditory neuropathy, or auditory dyssynchrony is clinically characterized by a disorder in understanding words, even in patients with light or moderate hearing loss. It has been related to generalized neuropathies and neonatal risk factors, such as intensive care admission, hyperbilirubinemia, sepsis and hypoxia. After clinical suspicion, the diagnosis is confirmed by the presence of otoacoustic emissions associated with the absence of waves in auditory brainstem responses. The therapy remains controversial, with the following options: hearing aids fitting and follow-up therapy for the habilitation/rehabilitation of language. In cases of failure there are also reports of satisfactory results with cochlear implants.

Conclusions: This study emphasizes the importance of the recognition of auditory neuropathy by pediatricians, which is yet an underreported problem in Latin America.

Key-words: hearing loss, sensorineural; hearing tests; neonatal screening.
Instituição: Hospital Federal da Lagoa, Rio de Janeiro, RJ, Brasil ${ }^{1}$ Graduada em Medicina pela Universidade Federal do Rio de Janeiro (UFRJ); Residente de Otorrinolaringologia do Terceiro Ano do Hospital Federal da Lagoa, Rio de Janeiro, RJ, Brasil

Doutor em Medicina pelo Curso de Pós-graduação em Otorrinolaringologia da Universidade Federal de São Paulo (Unifesp); Professor Associado de Otorrinolaringologia da UFRJ e Médico do Hospital Federal da Lagoa, Rio de Janeiro, RJ, Brasil

\author{
Endereço para correspondência: \\ Marco Antonio de M. T. de Lima \\ Hospital Federal da Lagoa - Ambulatório de Otorrinolaringologia \\ Rua Jardim Botânico, 501 - Jardim Botânico \\ CEP 22470-050 - Rio de Janeiro/RJ \\ E-mail: marcoantonio@hucff.ufr..br \\ Conflito de interesse: nada a declarar
}

Recebido em: 5/11/2010

Aprovado em: 13/6/2011 


\section{Introdução}

A neuropatia auditiva (NA) foi descrita inicialmente em 1996 por Starr et al, sendo caracterizada clinicamente pela dificuldade de compreensão das palavras, principalmente em ambientes ruidosos, apesar de o paciente, na maioria dos casos, responder a estímulos sonoros ${ }^{(1)}$. Esse quadro tem especial importância na infância por comprometer o desenvolvimento da linguagem ${ }^{(2-5)}$. A suspeita dessa entidade por parte dos pais ou profissionais de saúde deve considerar a presença de fatores de risco e o quadro clínico compatível.

Após a suspeita clínica, a confirmação diagnóstica é feita por meio de exames audiométricos e eletrofisiológicos, sendo encontradas, no quadro típico, otoemissões acústicas (OEA) presentes e potencial evocado de tronco encefálico (PEATE) ausente ou alterado, fato que sugere preservação da função das células ciliadas externas associada a distúrbio da condução neural ${ }^{(1,2,6-8)}$.

Em revisão bibliográfica realizada na literatura latinoamericana, encontraram-se poucos artigos referentes ao assunto. Mesmo na literatura mundial, é tema que recebeu relevância apenas nos últimos anos, em especial nas revistas de Pediatria. Assim, o objetivo deste trabalho foi alertar quanto a uma doença descrita recentemente e ainda pouco divulgada entre os pediatras, discutir seus principais fatores de risco e características clínico-audiológicas, com intuito de incluí-la entre os diagnósticos diferenciais dos transtornos de aquisição ou desenvolvimento da linguagem, considerando as possibilidades terapêuticas.

\section{Revisão}

A NA foi descrita pela primeira vez em 1996, em um trabalho de Starr et al, que investigou dez pacientes com queixa de dificuldade de entendimento da fala, principalmente em ambientes ruidosos, com suspeita inicial de comprometimento de sistema nervoso central. Tal suspeita foi levantada pelo aparente paradoxo da presença das OEA associada à ausência ou a alterações significativas no PEATE $^{(1)}$. As OEA consistem em sons emitidos pela cóclea, gerados pela contração das células ciliadas externas e, desse modo, antecedem a formação do PEATE. Este, por sua vez, reflete a condução de potenciais de ação gerados pela cóclea, e transmitidos pelo VIII par craniano ao tronco encefálico. Assim, a presença das OEA com ausência do PEATE é sugestiva de algum distúrbio na transmissão do estímulo elétrico da cóclea para o nervo auditivo, no próprio nervo ou, então, no tronco encefálico e vias auditivas centrais, pois uma vez geradas as OEA, este seria o caminho esperado a ser seguido pelo estímulo sonoro ${ }^{(1,6,7,9-12)}$.

Estudos posteriores relataram a associação da NA com as seguintes condições: ataxia de Friedreich, neuropatia isquêmica-hipóxica, hidrocefalia, degeneração espinocerebelar, neuropatia de Charcot Marie Tooth e outras neuropatias hereditárias sensório-motoras. Desse modo, acredita-se que a NA possa fazer parte do espectro da neuropatia generalizada, embora também se descrevam casos isolados $^{(1,6,7,11,13-15)}$. Há relatos de que o acometimento auditivo pode preceder em muitos anos o aparecimento da neuropatia periférica ${ }^{(16)}$.

Questiona-se se os casos isolados corresponderiam a apenas NA em si ou à primeira manifestação de um quadro de neuropatia sistêmica. Identificou-se um local possível de mutação relacionado à ocorrência da NA, o gene MPZ. Esse gene defeituoso levaria a uma doença axonal e consequente dessincronia na transmissão do impulso nervoso ${ }^{(7,17)}$. Há descrição de outro possível sítio genético responsável pela NA, o gene OTOF, que codifica a proteína otoferina, cuja mutação ocasionaria defeito na fusão de vesículas sinápticas nas células ciliadas internas ${ }^{(17,18)}$.

A literatura sugere que as doenças do período neonatal, especialmente associadas ao parto pré-termo (hipóxia, sepse, necessidade de ventilação mecânica, hiperbilirrubinemia e hemorragia intracraniana) têm papel causal na $\mathrm{NA}^{(2,6,8,12,19,20)}$

Para o diagnóstico da NA, observa-se, além da presença de OEA e a ausência ou alterações significativas no PEATE, a ausência do reflexo estapédico ou acústico durante a realização da imitanciometria ${ }^{(1,6-8)}$. Em crianças maiores, na audiometria tonal e vocal, o mais característico é a perda auditiva neurossensorial bilateral, de graus variáveis, sendo o mais importante nesses casos a incompatibilidade do limiar tonal com a inteligibilidade das palavras, já que esta, em geral, encontra-se bastante comprometida ${ }^{(1,6,8,11,13,21)}$. Isso significa que, apesar de muitas vezes ouvirem os sons, as crianças podem ser incapazes de compreender seu significado ${ }^{(7)}$.

Dessa forma, ao surgir a suspeita da NA, deve ser feito o diagnóstico diferencial com outras doenças neurológicas, malformações de orelha interna ou regiões retrococleares e agenesia de VIII par craniano, uma vez que podem cursar com as mesmas características clínicas. Dessa maneira, a avaliação multidisciplinar da criança, incluindo ao menos o neuropediatra e o otorrinolaringologista, deve ser considerada para a elucidação diagnóstica ${ }^{(22)}$. 
Trata-se de doença bilateral, porém há relatos de unilateralidade de NA em alguns estudos ${ }^{(8,12,15,23)}$. Apesar de ter sido considerada inicialmente bastante rara, estima-se, hoje em dia, que a NA compreenda 7 a $17 \%$ de todas as crianças com perda auditiva permanente ${ }^{(12,14,17,22)}$. É possível ainda que tal prevalência esteja subestimada, uma vez que as OEA podem desaparecer ao longo do tempo devido à falta de estímulo trófico pelo nervo auditivo ou ao uso de próteses amplificadoras. Nesse último caso, as OEA desparecem por mecanismo de trauma acústico, impossibilitando o diagnóstico clínico da $\mathrm{NA}^{(22,24)}$.

Deve-se prestar atenção a algumas condições que podem prejudicar o diagnóstico correto. As doenças da orelha externa ou média podem impedir a detecção das OEA e do reflexo estapédico ${ }^{(6,10,14)}$. Para verificar a integridade dessas estruturas, deve ser realizada otoscopia minuciosa e teste da imitanciometria, sendo tais exames fundamentais antes da realização da OEA e do PEATE, uma vez que os resultados dos dois últimos não são confiáveis se houverem alterações localizadas na orelha externa ou média, podendo gerar resultados falsos ${ }^{(10)}$.

Os primeiros anos de vida são fundamentais para o desenvolvimento da linguagem, devendo-se priorizar o diagnóstico precoce da deficiência auditiva ${ }^{(2-5)}$. Os benefícios do diagnóstico precoce incluem melhor aceitação da prótese pela criança, no caso de indicação de aparelho de amplificação sonora; manutenção da integridade das vias auditivas periféricas e centrais, evitando degeneração pelo desuso; estimulação diferenciada durante o período crítico para o desenvolvimento da linguagem; estímulo às habilidades da comunicação ${ }^{(25,26)}$.

Com o objetivo de detectar o mais precocemente possível as perdas auditivas, criaram-se os programas de triagem auditiva, que incluem, na maioria das vezes, apenas o teste de $\mathrm{OEA}^{(27-29)}$. Foi demonstrado que, após implementação de um programa de triagem neonatal que combinava OEA com PEATE, a incidência de NA aumentou de 1,2 para 4,1\%, e a idade média ao diagnóstico caiu de 34 para 5,7 meses de idade ${ }^{(14)}$. Dessa forma, deve-se prestar atenção, em especial, nas crianças com fatores de risco, que a combinação desses exames pode diminuir os resultados falso-negativos para $\mathrm{NA}^{(6,12,15,30-32)}$. Para neonatos com fatores de risco para deficiência auditiva e, principalmente, aqueles que permaneceram em unidades de terapia intensiva, devido à maior ocorrência do espectro da NA, o Joint Committee on Infant Hearing recomenda a triagem utilizando o PEATE e considera este o único método apropriado para a triagem auditiva dessa população ${ }^{(33)}$. No Brasil, essa recomendação foi reforçada em 2010 pelo Comitê Multiprofissional de Saúde Auditiva (COMUSA). Trata-se de comitê multidisciplinar que agrega áreas de estudo da fonoaudiologia, otologia, otorrinolaringologia e pediatria, criado em 2007, com o objetivo de discutir e referendar ações voltadas para a saúde auditiva ${ }^{(34)}$. O Comitê Brasileiro sobre Perdas Auditivas na Infância (CBPAI), desde 1999, orienta que a triagem auditiva neonatal deva ser realizada preferencialmente antes da alta da maternidade e, para nascidos fora de ambiente hospitalar, até os três meses de idade ${ }^{(35)}$.

Ainda não há consenso em relação à abordagem terapêutica da NA, por se tratar de entidade descrita recentemente ${ }^{(17)}$. Alguns estudos demonstraram que a perda auditiva pode melhorar espontaneamente em uma pequena amostra de pacientes portadores da doença ${ }^{(6,23,25)}$. A utilização de aparelhos de amplificação sonora é questionável, pois muitos pacientes podem não obter nenhum benefício com os mesmos, já que o problema, em boa parte das vezes, não está relacionado à perda auditiva e, sim, à compreensão das palavras. Assim, supõe-se que os aparelhos amplificadores possam até mesmo aumentar a lesão coclear ${ }^{(7,8,11,24)}$. Porém, em alguns casos, apenas com o uso de aparelho de amplificação sonora individual associado a terapias de reabilitação da linguagem, obtiveramse resultados satisfatórios, com razoável entendimento e desenvolvimento da linguagem ${ }^{(2,8,23,25,36)}$.

O implante coclear é apontado como uma alternativa para reabilitação desses pacientes, observando-se um número crescente de publicações sobre o tema. Embora ainda não tenham sido publicadas séries extensas de casos, os resultados demonstraram boa resposta, com melhora dos níveis de percepção da fala ${ }^{(6,8,9,11,14,23,25,36)}$. Entretanto, alguns autores relatam que crianças com NA submetidas ao implante coclear apresentaram pior resultado no que se refere à percepção da fala, quando comparadas com crianças implantadas por outra indicação clínica ${ }^{(14,23)}$. As crianças implantadas antes dos três anos de idade obtiveram melhores resultados ${ }^{(37)}$, o que se deve, provavelmente, à plasticidade das vias auditivas, que é a habilidade das conexões neurais modificarem-se por crescimento de sinapses, produzindo estimulações adequadas. Tal processo limita-se rapidamente a partir dos seis anos, sendo este um período crítico para a indicação do implante ${ }^{(2)}$.

\section{Comentários e recomendações finais}

O diagnóstico precoce da perda auditiva na população neonatal tem sido objeto de vários estudos e investigações. A seleção de um protocolo adequado e específico de triagem é difícil, sendo tópico de discussão em muitos centros de pesquisa. A pesquisa das OEA faz parte da maioria dos protocolos por ser um exame não invasivo, rápido, de fácil 
aplicação e com acurácia próxima de 100\%. Contudo, ao se utilizar esse teste como único método de triagem, apenas a integridade de parte da cóclea é avaliada e, portanto, deixam de serem diagnosticados os pacientes que apresentam outras possíveis causas de perda auditiva. Desse modo, apesar de sua importância fundamental no diagnóstico da NA, a OEA, como método isolado, não é capaz de detectar essa doença. Assim, o PEATE assume um papel fundamental na triagem auditiva, principalmente nas crianças com os fatores de risco já identificados como associados à NA. Para resultados mais confiáveis, na maioria das vezes, há necessidade de sedação das crianças não colaborativas. Devem ser excluídos, antes de sua realização, os fatores que podem prejudicar os resultados como, por exemplo, a presença de cerume em meato acústico externo ou otite média secretora. Atenção aos fatores de risco e políticas de triagem baseadas na realização de PEATE em neonatos com indicadores de

\section{Referências bibliográficas}

1. Starr A, Picton TW, Sininger Y, Hood LJ, Berlin Cl. Auditory neuropathy. Brain 1996;119:741-53.

2. Silva RC, Araújo SG. The results of cochlear implants in children with Auditory Neuropathy: literature review. Rev Soc Bras Fonoaudiol 2007;12:252-7.

3. Cristobal R, Oghalai JS. Hearing loss in children with very low birth weight: current review of epidemiology and pathophysiology. Arch Dis Child Fetal Neonatal Ed 2008;93:F462-8.

4. Silveira JA, Silveira AM, Bento RF, Butugan O, Miniti A, Almeida ER. Potenciais evocados auditivos (EcoG e/ou BERA) em 2545 crianças com suspeita de disacusia e/ou distúrbios de comunicação (estudo da etiologia, graus da deficiência auditiva e precocidade do diagnóstico). Rev Bras Otorrinolaringol 1996;62:388-408.

5. Casali RL, Santos MF. Auditory Brainstem Evoked Response: response patterns of full-term and premature infants. Braz J Otorhinolaryngol 2010;76:729-38.

6. Madden C, Rutter M, Hilbert L, Greinwald JH Jr, Choo DI. Clinical and audiological features in auditory neuropathy. Arch Otolaryngol Head Neck Surg 2002;128:1026-30.

7. Starr A, Michalewski HJ, Zeng FG, Fujikawa-Brooks S, Linthicum F, Kim CS et al. Pathology and physiology of auditory neuropathy with a novel mutation in the MPZ gene (Tyr145->Ser). Brain 2003;126:1604-19.

8. Dowley AC, Whitehouse WP, Mason SM, Cope Y, Grant J, Gibbin KP. Auditory neuropathy: unexpectedly common in a screened newborn population. Dev Med Child Neurol 2009;51:642-6.

9. Rapin I, Gravel J. "Auditory neuropathy": physiologic and pathologic evidence calls for more diagnostic specificity. Int J Pediatr Otorhinolaryngol 2003;67:707-28.

10. Berlin $\mathrm{Cl}$, Morlet T, Hood LJ. Auditory neuropathy/dyssynchrony: its diagnosis and management. Pediatr Clin North Am 2003;50:331-40.

11. Zeng FG, Kong YY, Michalewsky HJ, Starr A. Perceptual consequences of disrupted auditory nerve activity. J Neurophysiol 2005;93:3050-63.

12. Ngo RY, Tan HK, Balakrishnan A, Lim SB, Lazaroo DT. Auditory neuropathy/ auditory dys-synchrony detected by universal newborn hearing screening. Int J Pediatr Otorhinolaryngol 2006;70:1299-306.

13. Rance G, Corben LA, Du Bourg E, King A, Delatycki MB. Successful treatment of auditory perceptual disorder in individuals with Friedrich ataxia. Neuroscience 2010;171:552-5. risco para perda auditiva são necessárias para reduzir os erros diagnósticos dos transtornos auditivos na infância.

Com base na revisão da literatura realizada, concluí-se que o método de triagem auditiva limitado apenas à pesquisa das otoemissões acústicas ("teste da orelhinha"), quando realizado isoladamente, pode gerar uma falsa tranquilidade em profissionais de saúde e pais de crianças portadoras de NA, retardando o diagnóstico e acarretando as tão temidas consequências de uma intervenção tardia no que se refere à reabilitação auditiva.

O conhecimento da NA pelos pediatras torna-se ainda mais necessário quando se sabe que sua incidência tende a aumentar no futuro devido, por um lado, a técnicas melhores de detecção e, por outro, à redução nos índices de mortalidade por prematuridade ou baixo peso ao nascer, integrando o quadro de consequências neurológicas adversas resultantes do aumento da sobrevida de neonatos de alto risco.
14. Kirkim G, Serbetcioglu B, Erdag TK, Ceryan K. The frequency of auditory neuropathy detected by universal newborn hearing screening program. Int $J$ Pediatr Otorhinolaryngol 2008;72:1461-9.

15. Sanyelbhaa Talaat $H$, Kabel AH, Samy $H$, Elbadry M. Prevalence of auditory neuropathy (AN) among infants and young children with severe to profound hearing loss. Int J Pediatr Otorhinolaryngol 2009;73:937-9.

16. Spinelli M, Fávero-Breuel ML, Silva CM. Auditory neuropathy: clinical, diagnostic and therapeutic aspects. Rev Bras Otorrinolaringol 2001;67:863-7.

17. Roush P. Auditory neuropathy spectrum disorder: evaluation and management. Hearing J 2008;61:36-41.

18. Fávero ML, Romanos J, Mingroni-Netto RC, Balieiro CR, Donini TS, Spinelli M. Auditory Neuropathy Due to Mutations in OTOF Gene. Arch Otorhinolaryngol 2005;9:325-30.

19. Robertson CM, Howarth TM, Bork DL, Dinu IA. Permanent bilateral sensory and neural hearing loss of children after neonatal intensive care because of extreme prematurity: a thirty-year study. Pediatrics 2009;123:797-807.

20. Botelho FA, Bouzada MC, Resende LM, Silva CF, Oliveira EA. Prevalence of hearing impairment in children at risk. Braz J Otorhinolaryngol 2010;76:739-44.

21. Parra VM, Matas CG, Neves IF. Auditory neuropathy: a study case. Rev Bras Otorrinolaringol 2003;69:283-8.

22. Rance G. Auditory neuropathy/dys-synchrony and its perceptual consequences Trends Amplif 2005;9:1-43.

23. Mohd Khairi MD, Normastura AR, Wan Zaharah AW. Auditory neuropathy: three cases among a group with sensorioneural hearing loss. Singapore Med J 2009;50:324-5.

24. Tang TP, McPherson B, Yuen KC, Wong LL, Lee JS. Auditory neuropathy/ auditory dys-synchrony in school children with hearing loss: frequency of occurrence. Int J Pediatr Otorhinolaryngol 2004;68:175-83.

25. Berlin CI, Hood LJ, Morlet T, Wilensky D, Li L, Mattingly KR et al. Multisite diagnosis and management of 260 patients with auditory neuropathy/ dys-syncrhony (auditory neuropathy spectrum disorder). Int J Audiol 2010;49:30-43.

26. Durieux-Smith A, Fitzpatrick E, Whittingham J. Universal newborn hearing screening: a question of evidence. Int J Audiol 2008;47:1-10. 
27. Chapchap MJ, Segre CM. Universal newborn hearing screening and transient evoked otoacoustic emission: new concepts in Brazil. Scand Audiol Suppl 2001:33-6.

28. Baroch KA. Universal newborn hearing screening: fine-tuning the process. Curr Opin Otolaryngol Head Neck Surg 2003;11:424-7.

29. Pádua FG, Marone S, Bento RF, Carvallo RM, Durante AS, Soares JC et al. Newborn Hearing Screening Program: a challenge for its implantation. Arq Otorrinolangol 2005;9:190-4.

30. Gatanu - Grupo de apoio à triagem auditiva neonatal universal [homepage on the Internet]. São Paulo [cited 2010 Oct 20] Available from: http://www.gatanu. org/tan/protocolos.php

31. Joint Committee on Infant Hearing. Year 2000 position statement: principles and guidelines for early hearing detection and intervention programs. Am J Audiol 2000;9:9-29.

32. Butugan O, Santoro PP, Almeida ER, Silveira JA, Grasel SS. Early diangnosis of hearing loss in high-risk infants using auditory brainstem respons. Pediatria (São Paulo) 2000;22:115-22.

33. Joint Committee on Infant Hearing. Year 2007 Position Statement: principles and guidelines for early hearing detection and intervention programs. Pediatrics 2007;120:898-921.

34. Lewis DR, Marone SA, Mendes BC, Cruz OL, Nóbrega M. Multiprofessional committee on auditory health - COMUSA. Braz J Otorhinolaringol 2010;76:121-8.

35. Zocoli AMF, Riechel FC, Zeigelboim BS, Marques, JM. Hearing: a pediatrician's approach. Braz J Otorhinolaringol 2006;72:617-23.

36. Rance G, Barker EJ. Speech perception in children with auditory neuropathy/ dyssynchrony managed with either hearing AIDS or cochlear implants. Otol Neurotol 2008;29:179-82.

37. Kim LS, Jeong SW, Lee YW, Kim JS. Cochlear implantation in children. Auris Nasus Larynx 2010;37:6-17. 